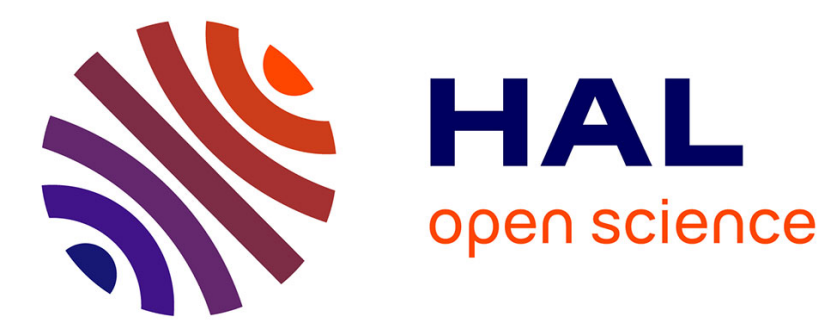

\title{
Quelle territorialité pour l'économie circulaire ? Interprétation des typologies de proximité dans la gestion des déchets
}

\author{
Jean-Baptiste Bahers, Mathieu Durand, Hélène Béraud
}

\section{To cite this version:}

Jean-Baptiste Bahers, Mathieu Durand, Hélène Béraud. Quelle territorialité pour l'économie circulaire ? Interprétation des typologies de proximité dans la gestion des déchets. Flux - Cahiers scientifiques internationaux Réseaux et territoires, 2017, Le retour de la proximité !, 109-110 (3), pp.129-141. 10.3917/flux1.109.0129 . hal-01662440

\section{HAL Id: hal-01662440 https://hal.science/hal-01662440}

Submitted on 26 Nov 2019

HAL is a multi-disciplinary open access archive for the deposit and dissemination of scientific research documents, whether they are published or not. The documents may come from teaching and research institutions in France or abroad, or from public or private research centers.
L'archive ouverte pluridisciplinaire HAL, est destinée au dépôt et à la diffusion de documents scientifiques de niveau recherche, publiés ou non, émanant des établissements d'enseignement et de recherche français ou étrangers, des laboratoires publics ou privés. 


\title{
Quelle territorialité pour l'économie circulaire? Interprétation des typologies de proximité dans la gestion des déchets
}

\author{
Jean-Baptiste Bahers \\ Mathieu Durand \\ Hélène Beraud
}

I 'économie circulaire, concept structurant aujourd'hui le Ldébat public autour des questions de maîtrise des flux de matière dans le cadre de la transition socio-écologique, n'est pas encore totalement définie scientifiquement (Buclet, 2015 ; Arnsperger, Bourg, 2016). L'un des aspects faisant notamment l'objet d'une absence de maîtrise est la question de sa territorialité. L'économie circulaire peut-elle être considérée à l'échelle planétaire, ou doit-elle nécessairement être mise en œuvre à l'échelle locale ? Si cette question n'est pas encore posée publiquement, elle fait déjà l'objet de positions bien tranchées chez les acteurs du domaine. L'une des façons de comprendre les enjeux de la territorialité de l'économie circulaire est de cibler l'un des trois grands domaines qui la composent (1), le plus ancien et le plus structuré : la gestion des déchets (2).

L'objectif de la gestion des déchets a pendant très longtemps été de les reléguer au plus loin des villes (Gouhier, 2000 ; Berdier, Deleuil, 2010 ; De Oliveira, 2013). Aujourd'hui, les logiques spatiales se complexifient, avec notamment I'obligation d'appliquer un "principe de proximité » (loi sur les déchets de 1992). Pour autant, rien n'indique ni l'échelle ni les modalités de cette proximité. Elle semble être à géométrie très variable en fonction du type $d^{\prime}$ acteur qui la prend en charge, du type de déchets et de la structuration des filières. L'objectif affiché à cette proximité est environnemental (limiter les exportations de nuisances), mais aussi socio-économique (tirer profit de matières premières de récupération, de matières premières urbaines (3) pour dynamiser une économie locale).
Cet article cherchera donc à comprendre l'opérationnalisation du principe de proximité en fonction des acteurs de la gestion des déchets, afin d'analyser la façon dont l'économie circulaire doit chercher à se l'approprier. Pour cela, il détaillera le cadre théorique qui sous-tend le développement de la "proximité » ainsi que la méthodologie utilisée pour comprendre son degré d'application. Ensuite, il proposera une analyse de l'interprétation de ce principe par les professionnels du secteur afin, en troisième section, de constituer une typologie de la proximité (4).

\section{LES ENJEUX DE LA DIMENSION TERRITORIALE DE L'ÉCONOMIE CIRCULAIRE}

Le concept d'économie circulaire fut importé par Dominique Bourg en France dans le cadre d'un doctorat sur le développement de ce sujet en Chine (Fan, Bourg, Erkman, 2006). L'acte de naissance politique de cette idée se situe dans les ateliers du Grenelle de l'Environnement de 2007 (5), confirmé par la conférence environnementale de 2013, et dont la définition officielle est inscrite dans la loi sur la transition énergétique en 2015. La dimension territoriale de l'économie circulaire est alors évoquée dans ce texte afin d'inviter à « la coopération entre acteurs économiques à l'échelle territoriale pertinente dans le respect du principe de proximité [soulignés par nous] » (6). Pour autant, aucune mention n'est faite de l'échelle territoriale à laquelle les flux de matière (au premier rang desquels les matières premières de récupération) devaient être maîtrisés. Pour l'Union Européenne, la dimension territoriale disparaît complètement 
au profit de l'efficience dans l'utilisation des ressources de manière à maximiser la croissance économique européenne (7) (8). On peut alors placer l'économie circulaire dans un cadre scientifique plus large, afin d'interroger les enjeux territoriaux sous-jacents à cette notion (Durand et al., 2015).

\section{Un débat ancien sur l'autonomie territoriale}

En parallèle à l'économie circulaire, il existe un second concept avec lequel les frontières sont souvent floues, celui d'écologie industrielle et territoriale, parfois compris dans une acceptation opérationnelle (la mise en œuvre de symbioses industrielles) ou dans un registre de champ scientifique (Junqua, Brullot, 2015). La première est pour les définitions officielles françaises incluses dans l'économie circulaire, comme une stratégie d'application. La seconde acceptation concerne des travaux scientifiques ayant cherché à le structurer depuis les années 1970, qui montrent au contraire que l'économie circulaire fait doublon avec cette notion (Buclet, 2015). Sans entrer ici dans le détail de ces superpositions conceptuelles, nous noterons simplement que la dimension territoriale de l'écologie industrielle est, elle, clairement identifiée. C'est d'ailleurs ce qui a valu, dans la littérature française, I'ajout du qualificatif de «territoriale » dans les années 2000. Cette volonté de maîtrise locale des flux entre en résonance avec l'idée d'autosuffisance territoriale, introduite par les travaux pionniers ayant par la suite donné naissance à l'idée de ville durable (Morris, 1982). Emelianoff (2007) relate que les premières réflexions sur la ville durable cherchaient à «stimuler la capacité à satisfaire localement les besoins fondamentaux » et à ne pas exporter les coûts de développement. L'idée était alors d'organiser des communautés tendant vers l'autosuffisance en termes d'approvisionnements en matières premières, notamment alimentaires et énergétiques. Il s'agissait également de ne pas aller au-delà de la capacité de charge de l'environnement en termes de réception de rejets émis par la ville. La mise en œuvre de ce concept sur un territoire limité dans sa dimension spatiale permet d'en maîtriser l'ensemble des enjeux et des interactions complexes. L'hinterland qui s'organise autour de la ville se limite alors à cet espace offrant une capacité de charge théorique suffisante. Ainsi, le territoire urbain serait en capacité de s'approvisionner et de se défaire de ses rejets en s'appuyant sur son environnement naturel et rural proche. Au-delà de la facilité fonctionnelle à travailler à l'échelle d'une ville, une délimitation artificielle de l'espace telle qu'imaginée ici, permet de maîtriser les flux et les acteurs de façon raisonnée.
Cependant, cette première vision de la ville durable comme structuration territoriale délimitée des flux de matières révèle rapidement des limites en mettant de côté la complexité des interdépendances à toutes les échelles. Ainsi, les logiques économico-commerciales visant à développer un libre marché s'opposent de façon frontale à toute tentative de limitation de la capacité d'échange de matière. Ces idées vont donc à I'encontre de toute tentative de contrainte territoriale. Ensuite, l'idée de sécurisation des approvisionnements alimentaires, consolidée depuis le XIX ${ }^{e}$ siècle, impose une insertion directe dans les flux internationaux, notamment pour les matières alimentaires. Enfin, la spécialisation technologique permettant de produire des biens de consommation ou de traiter des déchets spécifiques est contrainte à des économies d'échelle. L'ensemble de ces aspects rend assez rapidement caduque l'idée d'autosuffisance territoriale complète et amène à une gestion a-territoriale des flux.

Pourtant, la prise en compte de la proximité dans les flux de matières semble prendre de l'importance face à l'affirmation de nouveaux enjeux sociaux et environnementaux, revenant ainsi aux définitions initiales de la ville durable. La dynamique nouvelle de mise en place de circuits courts alimentaires ou d'agro-écologie permet de promouvoir la notion de proximité dans les flux de matières (par exemple, avec l'idée de «perma-circularité »-Arnsperger, Bourg, 2016). Les acteurs locaux ont donc souvent un regard bienveillant sur l'économie circulaire, qui privilégie le recyclage et la valorisation des déchets. D'une part, celle-ci permet de limiter l'impact potentiellement négatif des déchets sur leur environnement (limitation des transports de matières nuisibles, limitation des émissions de gaz à effet de serre, limitation du prélèvement de matières premières, etc.). D'autre part, elle favorise un impact positif sur I'environnement, à travers l'utilisation locale de matières de récupération, à l'opposé de l'enfouissement.

Par ailleurs, la recherche de conséquences socio-économiques positives est toujours plus prisée grâce à la création d'emplois que peut engendrer une économie locale dynamique s'appuyant en partie sur ces matières de récupération et sur l'activité du recyclage et du réemploi. L'enjeu socio-économique revêt une importance d'autant plus grande que la gestion de la proximité permet de tirer profit des déchets réutilisables et recyclables pour tenter de structurer une économie locale et de créer des emplois non délocalisables. En effet, il ne s'agit pas seulement de traiter les déchets à proximité, mais également 
de permettre à des activités industrielles et agricoles proches des villes de se développer pour transformer et utiliser cette matière de récupération, qualifiée de "matière secondaire » ou de «matière première urbaine » (Barles, 2005). La ville est alors perçue comme une grande consommatrice de ressources qu'elle ne puise aujourd'hui plus localement. Ces matériaux pourraient très bien être fournis par les filières locales de valorisation des déchets.

Ces questions de recherche, qui interrogent la proximité dans la gestion des matières premières urbaines et leur mise en valeur locale, restent pour le moment très peu abordées : "Dans ce sens, il est possible d'interroger le concept de proximité spatiale et sociale; la gouvernance des flux, impliquant le rôle des modes de vie et pratiques urbaines dans l'échange de matières; et le rôle des acteurs locaux et territoriaux. À ce jour, ce champ de recherche est incomplet » (9) (Barles, 2010, p. 452). Depuis, certains auteurs, notamment dans la littérature en écologie industrielle et territoriale, ont analysé le rôle de la proximité dans des démarches de symbioses industrielles (Beaurain, Brullot, 2011 ; Buclet, 2011), au sein de territoires portuaires (Shiller et al., 2014) ou d'un programme national d'échange de déchets et ressources au Royaume-Uni (Jensen et al., 2011). Il s'agit dans ces travaux de comprendre quels types de proximités (géographiques, organisationnelles ou institutionnelles) favorisent et facilitent les coopérations économiques entre acteurs, notamment au travers d'échanges de marchandises. Au contraire, Gregson et al. (2015) utilisent notamment la proximité spatiale pour faire la critique de ces démarches incapables de garder tous leurs déchets sur leur territoire et de les transformer en ressources. L'économie circulaire selon ces auteurs est donc une extension morale du marché des déchets soutenue par des discours de modernisation écologique. La remise en cause des grands réseaux urbains tend également à promouvoir des « écocycles urbains durables » reposant sur des cycles courts, la relocalisation du métabolisme urbain et une volonté d'autonomie (Coutard, 2010).

\section{Antériorité de la dimension territoriale dans la gestion des déchets}

Si la dimension territoriale de l'économie circulaire n'est à ce stade pas appréhendée, elle l'est de façon plus ancienne pour la gestion des déchets, l'une des composantes de la première notion. La politique de gestion des déchets prône en effet depuis les années 1990 un rapprochement des territoires de pro- duction des déchets avec les territoires de traitement, au nom du principe de proximité. Ce principe est un élément majeur du processus de territorialisation de la politique des déchets. II est mobilisé avec plusieurs objectifs.

Il apparaît dans la loi sur les déchets de 1992 (10) dans un objectif de limitation du transport des déchets et de leurs risques inhérents. Ce texte impose la réalisation de plans à l'échelle départementale pour les déchets non dangereux et à l'échelle régionale pour les déchets dangereux. Ces plans ont pour objectifs d'organiser et de planifier le traitement des déchets à ces deux échelles. "Chaque département [ou région dans le cas de l'île-de-France] doit prévoir une forte autonomie territoriale en matière d'installations de traitement. Le "tourisme des déchets", fréquemment dénoncé tant à un niveau international, que transfrontalier ou national, devrait dès lors constituer une exception»(Buclet, 2012, p. 67). Outre les aspects environnementaux, invoquer le principe de proximité permet également de définir " un territoire d'appartenance, et implicitement un territoire d'acceptation des nuisances résultant de l'élimination de "nos" déchets " (Rocher, 2006). Il s'agit alors de responsabiliser les citoyens à la gestion de leurs déchets, la proximité permettant leur appropriation (Méry, 2009 ; Nicourt, Girault, 2006 ; Rocher, 2006, 2008). L'enjeu de la légitimité dans les discours des acteurs peut également être central pour comprendre l'insertion territoriale des infrastructures de traitement des déchets (Matias Mendes, 2015). Les échelles départementales et régionales n'apparaissent alors plus comme les mieux adaptées à la mise en place de cette proximité de gestion. "On lui préfère des formes plus souples telles que des "bassins d'activités", des "communautés de destin" (CGP, 2003) ou encore des territoires intermédiaires (ADEME, 2005) » (Rocher, 2008). Depuis quelques années, le déchet n'est de nouveau (11) plus seulement vu comme une nuisance, mais comme une ressource (Bahers, Capurso, Gossart, 2015; D'Arras, 2008). Une gestion de proximité permettrait ainsi de réinjecter dans l'économie locale les déchets produits sur le territoire via des filières de recyclage.

Plusieurs visions de la proximité en fonction du type de déchets, de la filière de gestion ou de l'échelle d'étude coexistent. Des considérations économiques, de gouvernance sont à prendre en compte dans la localisation des sites de traitement. Ce choix dépasse les seules questions de gouvernance et les enjeux de proximité. II est lié à des logiques économiques (économie d'échelles, concurrence) ou techniques (procédés 
spécifiques pour certains déchets). Certains travaux ont aussi mobilisé la grille d'analyse socio-économique de la proximité (12), notamment les travaux de Bazin, Beckerich, Delaplace (2009). Ces derniers, concernant l'étude des filières de DEEE (Déchets d'Equipements Electriques et Electroniques) et VHU (Véhicules Hors d'Usage) en France, ont ainsi montré que l'organisation spatiale des activités dites de collecte de ces déchets privilégie la "proximité géographique des marchés " (Bazin, Beckerich, Delaplace, 2009), de même que les activités de démantèlement/déconstruction qui nécessitent une maind'œuvre importante et peu qualifiée. En revanche, les activités de traitement spécifique des ĐES et $\mathrm{VHU}$, une fois triés et regroupés, s'appuient sur « la proximité organisée [...] sur des lieux différents répondant à une logique de concentration de flux de matières devenues homogènes grâce au tri " (Bazin, Beckerich, Delaplace, 2009). En effet, ces entreprises s'affranchissent plus facilement des contraintes logistiques, car c'est la spécificité de l'outil et la performance de la transformation qui influent sur la circulation des flux. Aujourd'hui, le législateur ne définit pas précisément la proximité. La directive européenne de 2008 sur les déchets déclare que « le traitement des déchets doit permettre l'élimination ou la valorisation des déchets dans I'une des installations appropriées "les plus proches" »(Directive 2008/98/CE, article 16). L'article L541-1 du Code de I'Environnement affirme qu'il faut " organiser le transport des déchets et le limiter en distance et en volume ». Aucune information n'est donc donnée sur l'échelle territoriale à laquelle s'applique ce principe (Méry,

Enfin, les filières à Responsabilité Elargie du Producteur qui sont organisées par les professionnels, et non plus par les collectivités, prennent peu en compte le principe de proximité (Bahers, 2014). Les éco-organismes s'affranchissent souvent de cette question spatiale et sociale. Ils établissent des contrats avec des prestataires logistiques et de traitement choisis seulement selon des critères de respect de la réglementation et de coûts de transport et de traitement. Les filières s'organisent donc autour de grands pôles de traitement sans lien avec les échelles de planification, et sans prendre en compte les spécificités territoriales.

Cette planification territoriale évolue d'ailleurs. Après avoir été essentiellement mise en œuvre à l'échelle départementale (pour les déchets ménagers et les déchets du bâtiment et des travaux publics, alors que la gestion des déchets dangereux est planifiée à l'échelle régionale), elle va maintenant être organisée à l'échelle des nouvelles régions. Ceci s'inscrit dans la réforme territoriale à l'œuvre aujourd'hui, amenant la création de grandes régions et le renforcement généralisé de leurs prérogatives. C'est le résultat de la loi NOTRe (Nouvelle organisation territoriale de la République), publiée en août 2015. La Région est maintenant le territoire en charge de l'ensemble de la planification territoriale. L'objectif est multiple. II s'agit d'une part, de mettre en cohérence les différents documents de planification (économique, énergétique, environnementale, des transports, etc.) en les réalisant à une même échelle et dans un processus concerté. II s'agit d'autre part, concernant les déchets, de reconnaître que l'échelle départementale n'était plus adaptée à la diversification des filières, à la multiplication des acteurs, à l'agrandissement des aires de ehalandises des centres de stockage des déchets et aux questionnements récurrents autour de la valorisation énergétique.

\section{Méthodologie : quatre approches pour étudier la proximité}

Afin d'appréhender la façon dont le principe de proximité est appliqué en France, il est nécessaire de mieux connaître les flux de déchets circulant sur le territoire. Des méthodologies complexes permettent alors d'appréhender le métabolisme d'un territoire donné afin d'identifier les exutoires des déchets et la matière première de récupération approvisionnant l'économie.

Le texte présenté ici ne s'attarde pas sur les flux de déchets (13), mais la perception de la notion de proximité chez les acteurs des déchets. L'objectif est de croiser le discours des acteurs du territoire avec les politiques publiques menées, ainsi qu'avec les pratiques des entreprises et des organismes impliqués. Au-delà de la construction d'indicateurs quantitatifs spatialisés correspondant à l'analyse des flux, les résultats de cette recherche résident dans l'analyse empirique de l'opérationnalisation d'une économie circulaire locale dans les plans d'action publique et les pratiques des acteurs. Les interrogations sont par exemple nombreuses sur la façon dont la planification territoriale de la gestion des déchets permet de prendre en compte la notion de proximité des échanges de matières. Pour cela, 57 entretiens (14) semi-directifs ont été réalisés entre 2013 et 2015, avec des gestionnaires de déchets (collectivités locales ou entreprises), des industriels (recyclage ou élimination), des éco-organismes, les services de l'État, des instances en charge de la planification (départements, régions, observatoires) ou encore des associations et des observateurs. 


\section{UNE PERCEPTION VARIABLE DE LA NOTION DE PROXIMITÉ}

Le principe de proximité s'applique avec une géométrie variable en fonction des types de déchets (Durand, Bahers, Beraud, 2016). Certains sont pour l'essentiel gérés localement comme les putrescibles, les pondéreux ou les déchets ultimes. D'autres ont tendance à parcourir de grandes distances tels que les déchets dangereux, ceux à forte valeur ajoutée, ou faisant I'objet de filières de Responsabilité Elargie du Producteur (15). Quelles que soient les logiques spatiales d'application de ce principe de proximité, on retrouve toujours des logiques similaires. La proximité y apparaît alors selon une typologie complexe, en fonction de critères propres à chaque acteur, chaque situation et chaque priorité.

La proximité peut être définie en fonction de plusieurs critères, privilégiés différemment en fonction des acteurs, des contextes et des types de déchets. Ces critères signifient qu'il y a en réalité plusieurs proximités qui peuvent être définies de façon distincte en fonction des contextes. La législation restant très floue dans le domaine, il est donc important de bien structurer cette typologie afin de comprendre la façon dont s'exprime cette proximité et éventuellement la façon dont on peut la gérer. La perception de la proximité est donc variable en fonction des acteurs des déchets impliqués et du contexte (16). Chacun va l'appréhender selon ses propres contraintes, techniques, économiques, en termes de communication ou d'enjeux de territoire.

\section{La proximité : une image de marque}

La proximité est tout d'abord une image de marque à faire valoir pour les entreprises et pour les collectivités locales : "La proximité, c'est l'image qu'on peut renvoyer et qu'on promeut » (Responsable de centre de recyclage, 2014). Elle s'exprime par les relations de confiance avec les riverains et par un ancrage territorial fort. Les entreprises ont ainsi la volonté de communiquer et de promouvoir cette dimension valorisante : "commercialement, on communique beaucoup sur le "site de proximité" [...] sur des marchés de proximité géographique, à l'heure des "bilans carbone", de la proximité environnementale-» (Directeur centre de traitement, 2015). Cette image est souvent associée à la dynamisation socio-économique du territoire : « la proximité, c'est l'image de la société. On veut faire bénéficier de la matière aux locaux et faciliter l'intégration de la société dans le paysage local » (Responsable centre de traitement, 2015).
Cette vision de la proximité, mise en avant par des gestionnaires de sites locaux (parfois des entreprises), s'oppose à la stratégie de développement national et international des grands groupes qui possèdent plusieurs sites. Ce sont essentiellement les entreprises qui n'ont qu'une seule exploitation, qui mobilisent cet argument. Ainsi, ils contournent la limite d'une unique implantation en démontrant leur ancrage local. Il s'agit d'une stratégie commerciale " par bon sens, conviction, d'une part pour économiser des coûts et pour l'impact carbone. C'est du marketing » (Responsable centre de traitement, 2015). Cette représentation de l'entreprise locale est donc importante pour valoriser son identité. C'est par exemple pour cela que la multinationale du déchet Sita s'est associée avec l'entreprise de récupération et de valorisation des déchets sarthoise Passenaud en 2012 (à parts égales) dans la création du nouveau centre du tri du Mans : Valor Pôle 72.

\section{Une meilleure acceptation sociale des infrastructures dans la proximité}

La période du NIMBY (Not in My Back Yard) a laissé des traces dans la culture des entreprises de traitement et elle n'est pas encore achevée. Ainsi, des dispositifs réglementaires ont été créés par les services de l'État, comme les enquêtes publiques et les Commissions Locales d'Information et de Surveillance (CLIS), devenues par la suite les Commissions de Suivi des Sites (CSS). Les entreprises ont appris à s'en accommoder et à construire un mécanisme d'acceptation sociale de leurs infrastructures, afin de transformer la contrainte liée à la proximité spatiale. Ce mécanisme se matérialise d'une part par des rencontres régulières, car «les relations avec les riverains, c'est maintenir le lien de proximité, on va les voir régulièrement » (Directeur centre de traitement, 2015). D'autre part, cette acceptation se traduit par des échanges plus fréquents plus ou moins positifs : «La proximité c'est à 150 mètres, ce sont nos voisins. Dès qu'il y a une mauvaise odeur, ils sont à la porte, ou pire à la mairie. La préfecture a mis en place un jury de nez et une réunion publique tous les trois mois. Les relations s'améliorent dès que les nuisances sont finies, enfin ça prend du temps » (Responsable centre de traitement, 2015). Ces industriels n'hésitent pas à utiliser ces dispositifs de concertation pour améliorer leurs relations avec les habitants riverains.

Enfin, certaines sociétés n'hésitent pas à co-construire des activités avec leurs riverains en subventionnant des associations environnementales locales pour le suivi des impacts industriels. Cela s'exprime par « une grande proximité avec les 
riverains en intégrant leurs compétences » (Responsable centre traitement, 2014). Malgré ces dispositifs, il existe toujours de nombreux freins sociaux à l'implantation de sites de traitement à proximité des habitations. Même si certains modèles économiques imposent des distances faibles entre installations et centres urbains comme pour les déchets du BTP par exemple, il y a "souvent des conflits avec les aménageurs et les élus qui souhaiteraient éloigner ces activités des centres urbains » (Ingénieur centre de recyclage de déchets du BTP, 2014).

\section{Les réseaux humains : quand la proximité engendre des marchés commerciaux}

La proximité s'exprime aussi par les interconnaissances entre professionnels des déchets. Les échanges et les partenariats sont ainsi facilités par le mouvement d'un collaborateur d'une société à une autre : "j'ai travaillé 11 ans chez $X$ avant d'être responsable d'agences chez $\mathrm{Y}$. Et mon principal client aval est $X$. Mes collectivités clientes chez $X$ sont devenues mes clients chez Y» (Responsable centre de recyclage, 2015). Quoiqu'étant dans une situation de concurrence souvent forte, les acteurs du déchet se connaissent à l'échelle d'un territoire : "Avec certains concurrents, on fonctionne à la complémentarité, à l'échange » (Responsable centre de recyclage, 2015). Les réseaux sociaux et professionnels structurent ainsi une proximité entre individus et entre institutions : «le monde est petit, tout le monde se connaît, ou au moins apprend à se connaître par un tiers, ou par le parcours professionnel » (Responsable centre de traitement, 2015). Certains réseaux ad hoc comme les réseaux d'entreprises, d'éco-activités ou d'écologie industrielle favorisent ce rapprochement. Les processus communs, tels que la construction de documents de planification territoriale à laquelle tous les acteurs du déchet sont conviés, invitent également à cette interconnaissance.

Cette proximité entre professionnels permet d'être plus réactifs sur les marchés, d'échanger de bonnes pratiques et de mutualiser des moyens. Il s'agit par exemple de répondre à des appels d'offres en consortium d'entreprises ou de créer une activité commune-comme le recyclage de matelas à Rennes - co-portée par une entreprise d'insertion et un groupe industriel. Le lien de confiance entre partenaires et clients est souvent mis en avant par les entreprises. Pour certains acteurs interrogés, privés comme publics, bâtir cette confiance semble être presque aussi important que d'être compétitifs sur les coûts de traitement. Ainsi, les nouvelles perspectives commerciales de l'économie circulaire seraient «du business écologique et économique, lié à la confiance entre clients plutôt que des relations sur le coût » (Responsable centre de traitement, 2015). II faut dès lors « entretenir surtout un lien important avec les partenaires [...] sur un maillon de la chaîne ou un autre » (Responsable centre de traitement, 2014). Cette relation de confiance se construit également sur des valeurs de proximité avec les partenaires, car certaines sociétés se revendiquent comme " une entreprise familiale et dont la richesse est située dans la proximité avec nos clients. » (Responsable entreprise de collecte des déchets, 2015). Si l'encadrement strict des marchés publics a largement assaini ce genre de relation entre prestataires et commanditaires, le lien humain reste un facteur important de collaboration. Cette relation interpersonnelle reste cependant largement contrainte par la concurrence économique $d^{\prime}$ 'une part, et les marchés publics d'autre part.

\section{La proximité comme une contrainte}

Si la proximité s'est construite comme une contrainte permettant de limiter les risques sanitaires et environnementaux, elle dévie progressivement vers une force permettant de développer une économie locale. II existe toutefois de nombreux freins avant d'atteindre une économie circulaire qui intègre ce critère de proximité. Malgré les contraintes économiques et environnementales du transport des déchets, les entreprises s'en affranchissent facilement lorsque les flux sont massifiés et à haute valeur ajoutée. Les entreprises connaissent bien ce paradoxe: "La proximité est indispensable pour toute typologie de déchets. Ensuite, plus on trie, moins elle est importante» (Responsable centre de traitement, 2014). Cette massification des flux conduit aussi à un mouvement de concentration de sociétés pour augmenter leur capacité d'investissement au détriment des "petits acteurs locaux [...] qui ont développé des savoir-faire locaux » (Responsable centre de traitement, 2014).

D'autre part, les longues distances à parcourir ne sont pas systématiquement synonymes de charges onéreuses et les entreprises trouvent des stratégies pour baisser ces coûts, comme la « reverse supply chain (logistique inverse) [...] qui permet un fret de retour pour avoir la capacité de reprendre un déchet localement [...] pour diviser le coût de transport par deux » (Responsable centre de recyclage, 2014). Enfin, toutes les sociétés ne militent pas pour une proximité du traitement, notamment les groupes qui possèdent des installations spécifiques : " la gestion de proximité n'est pas toujours acceptable économiquement [...] et il ne faut pas entraver la libre circulation des marchandises»(Directeur de la FNADE, 2014). 
La logique de marché reste le principal critère de gestion des déchets, qu'il s'agisse d'élimination ou de rachat des matières de récupération.

\section{TYPOLOGIE DE L'INTERPRÉTATION DE LA NOTION DE PROXIMITÉ}

Les idées évoquées dans la section précédente permettent de structurer plusieurs types de en fonction des acteurs, des déchets et des territoires. Certaines viennent compléter des typologies déjà largement établies par l'école de la proximité française (notamment : Gilly, Torre, 2000 ; Torre, Beuret, 2012). D'autres types de proximités sont spécifiques à l'étude de cas développée ici. La typologie établie ici peut également être croisée avec des registres plus larges, définis par d'autres auteurs concernant la perception des enjeux autour des déchets de la part des populations européennes (Cirelli, Maccaglia, 2015 ; Cirelli, Maccaglia, Melé, 2017). L’approche proposée ici reprend cette idée d'une typologie des proximités, mais en les appliquant à une entrée différente qui est celle de la distance parcourue par les déchets avant leur traitement final. II s'agit de s'appuyer sur le sens légal du principe de proximité tel que défini dans la législation française et européenne, afin, par la suite, de penser le territoire sur lequel s'applique l'extension conceptuelle de la gestion des déchets : l'économie circulaire.

Six types de proximités ont ainsi été identifiés (cf. figure ci-dessous).

\section{Typologie des proximités par rapport aux flux de déchets}

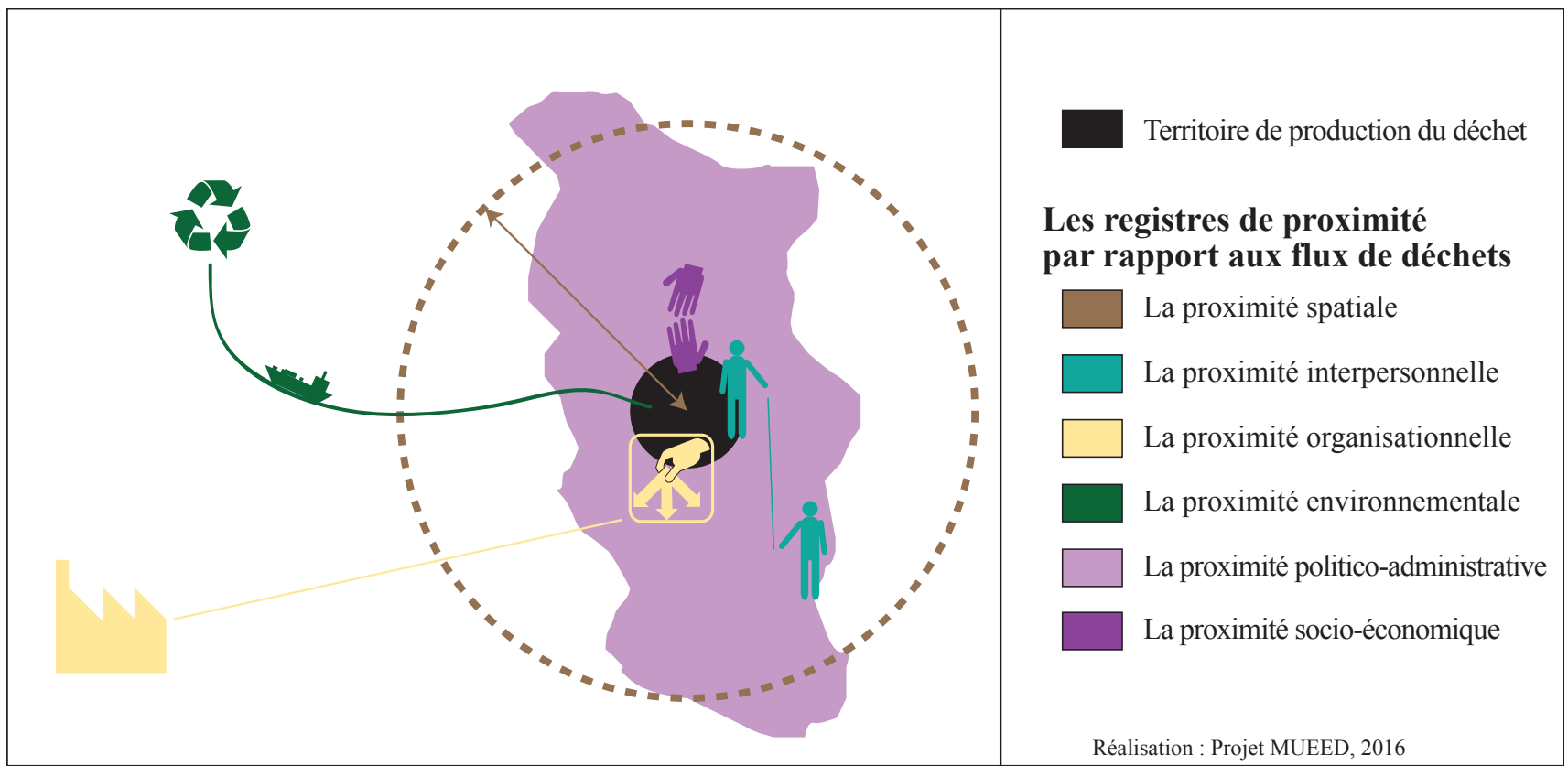

\section{La proximité spatiale : mesurer la distance}

La proximité spatiale permet de mesurer la distance (Gilly, Torre, 2000). Elle vise à identifier le parcours des flux (17) de déchets et en mesurer la distance parcourue. Il est alors, dans I'absolu, possible de définir un kilométrage précis parcouru par chaque type de déchets à partir de son lieu de production, pour estimer s'ils sont gérés dans une proximité définie. II ne s'agit là que d'une application basique du principe uniquement exprimé en nombre de kilomètres, éventuellement modulable selon les types de déchets (les déchets putrescibles par exemple gérés sous quelques kilomètres, alors que les métaux vont facilement parcourir des milliers de kilomètres).

\section{La proximité relationnelle : les relations humaines toujours présentes}

La proximité relationnelle correspond à l'appartenance à un réseau d'interconnaissance évoqué dans la section précédente, ainsi qu'à la similitude dans les cadres cognitifs (Torre, 
Beuret, 2012). Quoique n'ayant pas officiellement sa place dans les marchés publics et les appels d'offres de gestion des déchets (afin d'éviter tout risque de corruption ou de malversation), ces interrelations humaines tiennent toujours une certaine place dans la gestion des déchets selon les acteurs interrogés. Tout en s'inscrivant dans un cadre juridique et économique très contraint, la connaissance entre plusieurs personnes peut alors avoir un rôle dans le choix d'un exutoire final pour un déchet. Cette proximité n'est pas exclusive des relations commerciales entre acteurs professionnels, qui sont au contraire renforcées par ces échanges entre individus d'organisations différentes.

\section{La proximité industrielle : priorité aux enjeux techniques et d'économie d'échelle}

La proximité industrielle peut se décliner selon deux modalités. La première concerne la structuration des filières industrielles et entrepreneuriales. À l'échelle française, les entreprises du déchet sont en effet aujourd'hui regroupées en trois ou quatre grands consortiums (sous forme de filiales ou de partenariats stratégiques), ayant ainsi une maîtrise de l'ensemble du cycle, depuis la collecte jusqu'aux différents modes de traitement des déchets. Les centres de tri d'un grand groupe industriel vont généralement privilégier les entreprises de recyclage ou les centres d'enfouissement du même groupe ou d'entreprises avec lesquelles il existe un partenariat. II s'agit d'une logique économique. La proximité industrielle est ici structurée en fonction de logiques économico-institutionnelles, minimisant la simple distance entre deux installations pour privilégier le partenariat économique.

La proximité industrielle peut également être structurée par une filière technico-industrielle spécifique. Certains types de déchets ou de matériaux particuliers nécessitent en effet d'être envoyés vers un centre de démantèlement donné ou industrie de recyclage spécifique. Ce genre d'installation, en fonction de son coût (investissement ou fonctionnement), sa technicité et la faiblesse des gisements, peut être plus ou moins disséminé sur le territoire. Les déchets peuvent donc parfois avoir besoin de parcourir de grandes distances pour trouver l'installation techniquement adaptée à leur élimination/valorisation. La proximité industrielle amène alors souvent à négliger la proximité spatiale, puisqu'elle peut nécessiter des infrastructures très spécialisées (dans le recyclage de matériaux à forte valeur ajoutée ou l'élimination des déchets dangereux par exemple) et donc très éloignées.

\section{La proximité environnementale : la difficile mesure de l'impact environnemental}

La proximité environnementale est davantage basée sur l'optimisation des transports et du traitement final, en lien avec des méthodes d'évaluation environnementale comme le bilan carbone. Les analyses d'empreinte environnementale (18) réalisées sur les villes étudiées montrent que, contrairement à ce qui était imaginé initialement, il n'y a pas de lien systématique entre la proximité environnementale et la proximité spatiale. En effet, certains déchets peuvent parcourir plusieurs milliers de kilomètres pour être valorisés par une technologie qui permet de réduire l'impact environnemental global du matériau (par rapport à une extraction de matière première dans le milieu naturel). Au contraire, pour d'autres types de déchets, la proximité environnementale impose la proximité spatiale. Il faut dans tous les cas avoir connaissance de cet écart entre l'impact environnemental du transport et celui du traitement. Ce rapport s'exprime de façon très différente en fonction des types de déchets (19).

\section{La proximité politico-administrative : des contraintes très réglementées}

La proximité politique reprend la façon dont les acteurs publics s'emparent de cet enjeu, au titre du développement socio-économique et de la prise en compte des intérêts citoyens locaux. Cette proximité politique n'est pas forcément en lien avec les proximités évoquées précédemment. Elle a pourtant son importance, puisqu'elle est portée par des acteurs - représentant la population - influents dans l'élaboration des politiques locales de gestion des déchets. La proximité politique conduit à une meilleure acceptation des infrastructures nécessaires pour gérer les déchets. Elle permet également d'aller vers l'optimisation de la valorisation des déchets (notamment grâce au tri), mais surtout à la réduction des déchets. De nombreuses actions locales peuvent être menées sous l'effet d'une impulsion citoyenne.

La proximité politico-administrative a un pouvoir contraignant important, puisqu'elle s'appuie sur des juridictions aux compétences légalement définies. II s'agit en premier lieu des collectivités locales en charge de la gestion des déchets (collecte, transport et traitement). Qu'il s'agisse de Communautés de communes, de Communautés d'agglomération ou urbaines, ou de syndicats intercommunaux, leurs choix permettent, dans une certaine mesure, de mettre en application le principe de proximité. Si leur action est très cadrée par le code des marchés publics d'une part (qui interdit le favoritisme d'entreprises locales), par les conventions signées avec les éco-organismes 
d'autre part, elles peuvent tout de même tenter de privilégier les exutoires de proximité.

La seconde échelle territoriale en capacité de mettre en place une proximité politico-administrative, est le département et/ou la région. Il s'agit des instances en charge des différentes planifications territoriales de gestion des déchets. Comme indiqué précédemment, avec les récentes évolutions de structuration territoriale en France, avec la création de grandes régions et le renforcement généralisé de leurs prérogatives (20), les Régions récupèrent l'ensemble de la planification territoriale, hier, en grande partie, de la responsabilité des départements.

\section{La proximité socio-économique : volonté de développer le territoire local}

Le dernier type de proximité vise à stimuler le tissu socio-économique local grâce à l'exploitation des déchets. Qu'il s'agisse de collecte, de tri, de valorisation et même de prévention, les déchets permettent le développement d'une économie circulaire locale. Elle amène par exemple les producteurs de déchets (collectivités, mais aussi certaines entreprises) à mettre en avant une structure de l'économie sociale et solidaire connue et ancrée localement, et non une grande entreprise perçue comme éloignée du territoire. L'enjeu est la création locale d'emplois.

La bataille est alors intense dans certains domaines comme les Déchets d'Equipements Electriques et Electroniques (DES) par exemple. Les éco-organismes, structures agréées par l'État, mais pilotées par les entreprises productrices d'appareils électroniquesł, cherchent ainsi à capter de grands gisements de dans des usines de recyclage nationales. À l'inverse, les acteurs de l'économie sociale et solidaire, historiquement présents sur ce secteur et récemment concurrencés par l'émergence des éco-organismes, défendent leur implantation locale, souvent soutenus par les collectivités locales. La proximité socio-économique entre alors en concurrence avec la proximité industrielle. Chaque logique met ainsi en avant des représentations différenciées dans l'appropriation et l'utilisation du principe de proximité.

\section{CONCLUSION : QUEL TRANSFERT VERS L'ÉCONOMIE CIRCULAIRE?}

Nous l'avons évoqué en introduction de ce texte, la question des déchets est difficilement appréhendable de façon isolée aujourd'hui, puisque ces objets ne sont que le résultat du fonctionnement d'un système territorial. La notion d'économie circulaire les replace ainsi dans un cadre plus large. L'objet n'est pas d'entrer ici dans les débats relatifs à la pertinence de cette notion et à l'efficacité de sa définition (Ghisellik Eialini, Ulgiati, 2016; Desvaux, 2017). Il s'agit plutôt de partir du constat que dans toutes les définitions données, la dimension territoriale est très peu présente. Nous faisons donc le choix de nous restreindre ici à la définition officielle française de l'économie circulaire proposée par l'ADEME (2014), structurée autour de sept piliers (voir note (1)). Cette définition est différente de celle des promoteurs associatifs ou de l'Union Européenne (voir plus haut), mais I'ADEME est un acteur incontournable et prescripteur de dispositifs d'action sur les questions environnementales en France. Nous esquissons alors ici une interprétation possible de la typologie des proximités présentée précédemment, articulée avec chacun des autres piliers de l'économie circulaire.

L'approvisionnement durable en ressources, premier des sept piliers selon I'ADEME, ne mentionne pas de principe de proximité dans son acceptation. On peut considérer que les enjeux techniques et les économies d'échelles sont les principales logiques mises en avant. Ainsi, la proximité industrielle pourrait révéler cette stratégie où l'on privilégie les filières structurées. La proximité spatiale peut être importante dans I'approvisionnement en matériaux pondéreux ou putrescibles, tout en intégrant des critères environnementaux de limitation des transports et des impacts liés à l'exploitation des carrières. La proximité politico-administrative peut être interprétée dans le cadre des schémas régionaux des carrières (21).

Les approches dites "produit » comme l'éco-conception, qui *ise à réduire les impacts environnementaux tout au long du cycle de vie des produits, ne semblent a priori pas s'articuler à une dimension territoriale. Seule la proximité industrielle peut avoir un poids (quoique limité) dans les logiques des entreprises en faisant de la recherche et développement, vers des filières structurées pour optimiser les économies d'échelles.

L'écologie industrielle et territoriale est parfois considérée à elle seule comme étant la dimension territoriale de l'économie circulaire. La grille complète des proximités serait pertinente à mobiliser, avec selon les cas une place privilégiée pour la proximité spatiale (indispensable à toute mutualisation de flux) ou relationnelle et politico-administrative (afin de permettre une collaboration pérenne) (22).

L'économie de fonctionnalité, cherchant à remplacer la propriété d'un produit matériel par l'usage d'un service, s'inscrit clairement dans un territoire spatialement limité, dans une 
perspective de rapprochement entre fabricant et consommateur (Buclet, 2014). Ainsi, les proximités spatiales et relationnelles sont les plus à même de révéler la territorialité de ces démarches. L'économie de fonctionnalité a la particularité selon les initiatives de créer des échanges interpersonnels à l'échelle d'une ville ou d'un quartier, voire de favoriser la proximité socio-économique visant à dynamiser les acteurs locaux.

La consommation responsable vise en premier lieu à soutenir l'achat d'éco-produits et n'est pas identifiée en tant que telle dans une perspective de territorialisation dans la définition donnée par l'ADEME. C'est sans compter sur les circuits courts alimentaires (ou autres) qui font florès dans les discours institutionnels. Il faudrait probablement analyser cette stratégie sous l'angle de la proximité socio-économique pour montrer en quoi l'ancrage local est source de développement économique et de préservation des ressources.

L'allongement de la durée d'usage au travers du réemploi, de la réparation et de la réutilisation ne mentionne une nouvelle fois pas d'échelle territoriale adéquate pour la mettre en œuvre. Cependant, il est difficile d'imaginer que, sans proximité spatiale, cette stratégie soit efficiente d'un point de vue environnemental. C'est sans doute avec cette démarche que l'analyse des proximités socio-économiques, au travers d'un écosystème d'acteurs de l'économie sociale et solidaire, serait la plus intéressante à réaliser. Les enjeux résident notamment dans le développement d'une écologie « artisanale » et territoriale.

Les relations identifiées ici mériteront d'être développées individuellement dans des travaux à venir, afin d'avoir une vision plus globale des enjeux de la territorialité de l'économie circulaire. Il s'agira alors d'interroger les acteurs afin de comprendre leur logique d'appropriation. En effet, nous avons montré que pour la gestion des déchets en Europe, si la proximité est légalement l'un des principes de base, elle est pour autant laissée à la libre interprétation des acteurs du domaine, n'offrant ainsi aucun moyen réel de mise en application, ni même d'appréhension commune. Entre la nécessité de prendre en compte les riverains d'un centre d'enfouissement, le développement de l'économie sociale et solidaire locale, les logiques de grands groupes industriels ou encore la spécificité technique de l'élimination des déchets dangereux, le principe de proximité ne s'applique jamais à la même échelle. Des contraintes spécifiques aux types de déchets et à la position de chaque acteur en font une proximité à géométrie variable.
Intégrant la gestion des déchets dans l'acception plus large d'économie circulaire, il est important de clarifier les attentes territoriales sur cette dernière notion. Si l'application du principe de proximité dans la gestion des déchets est déjà imparfaite, est-ce que la simple mention d'une économie circulaire peut permettre de le renforcer ? Cette économie circulaire a-telle vocation à s'appliquer à l'échelle locale, ou peut-elle être, sur certains flux, considérée comme étant planétaire? Les impacts environnementaux relatifs aux distances parcourues par les flux de matières dépendent énormément de leur caractère pondéreux, putrescible, dangereux, etc. Pour autant, les enjeux socio-économiques sont en grande part présents aux échelles locales. Toutes les opérations amont au statut de déchets (écologie industrielle et territoriale, économie de fonctionnalité, prolongement de la durée de vie des produits...) ont vocation à interroger le caractère territorial de l'économie circulaire.

Jean-Baptiste Bahers est ingénieur et docteur en aménagement $d u$ territoire. Il est enseignant-chercheur en évaluation environnementale et écologie territoriale à l'École des Métiers de I'Environnement (EME) de Rennes. II est chercheur permanent au laboratoire ESO (Espaces et Sociétés) (UMR 6590). Ses travaux concernent la territorialisation des politiques européennes et françaises en matière d'économie circulaire, l'analyse des filières éco-industrielles et le développement de l'écologie industrielle et territoriale. Il s'intéresse également au développement théorique du champ du métabolisme urbain et territorial en France et en Europe. jeanbaptistebahers@ecole-eme.fr

Mathieu Durand est Maître de Conférences en Aménagement du territoire au Mans Université, chercheur au laboratoire ESO (Espaces et Sociétés) du CNRS (UMR 6590) et à I'IFEA (Institut Français d'Etudes Andines). Responsable du Master "Management et Ingénierie des Déchets - Économie Circulaire ». II mène des projets de recherche sur la spatialisation de la gestion des déchets en France, en lien avec l'ADEME, les collectivités locales et les entreprises. Il développe également des recherches en Amérique Latine, avec diverses universités et avec l'AFD, afin de comprendre en quoi un modèle low-tech peut s'avérer réplicable vers les pays du nord. Mathieu.Durand@univ-lemans.fr

Hélène Beraud est docteur en Génie urbain. Elle est Maître de au département Génie urbain de I'Université ParisEst Marne-La-Vallée et chercheur au Lab'Urba. Elle a soutenu sa thèse en 2013 sur la "Résilience du service de gestion des déchets aux inondations » au LEESU (Université Paris-Est). Ses travaux s'inscrivent dans le domaine de l'écologie territoriale et de la résilience, et portent sur le service de gestion des déchets et son rôle dans le fonctionnement des villes. helene.beraud@u-pem.fr 


\section{Notes}

(1) Selon la définition de l'ADEME, l'économie circulaire est composée de trois grands secteurs (eux-mêmes divisés en sept piliers) : I'offre des acteurs économiques (approvisionnement durable, éco-conception, écologie industrielle et territoriale, économie de fonctionnalité), la demande des consommateurs (consommation responsable, allongement de la durée de vie des produits) et la gestion des déchets (recyclage). Si cette définition est critiquée (Đerux, 2017), elle fait aujourd'hui figure de référence pour les acteurs opérationnels qui seront interrogés par la suite dans cet article.

(2) Un autre de ses domaines, bien antérieur dans sa définition, a également une dimension territoriale très marquée : I'Écologie Industrielle et Territoriale. II est cependant moins largement appliqué que la gestion des déchets.

(3) Le premier terme est utilisé par l'ADEME, le second par Barles (2005).

(4) Ce texte est issu du projet de recherche MUEED (Métabolisme Urbain, Empreinte Environnementale et politiques de gestion des Déchets), financé par le programme "Déchets et société" de l'ADEME et mené par l'Université du Maine (UMR ESO CNRS), l'École des Métiers de l'Environnement (UMR ESO CNRS), et I'Université de Paris-Est Marne-La-Vallée (Lab'urba, équipe Génie Urbain).

(5) En 2007, dans le rapport du groupe 6 « Promouvoir des modes de développement écologiques favorables à la compétitivité et à l'emploi » : «l'enjeu économique de la politique environnementale [...] qui fasse notamment plus de place à une économie circulaire, fondée sur la réduction et le recyclage des déchets, et plus généralement sur une utilisation plus efficace des ressources ».

(6) LOI n² 2015-992 du 17 août 2015 relative à la transition énergétique pour la croissance verte

(7) European Commission, 2014, The Circular Economy: connecting, creating and conserving value. [En ligne] (consulté le 25 septembre 2017) Disponible à l'adresse : http://ec.europa.eu/environment/circular-economy/

(8) European Commission, 2015, Closing the loop - An EU action plan for the Circular Economy. [En ligne] (consulté le 25 septembre 2017) Disponible à l'adresse : https://ec.europa. eu/priorities/jobs-growth-and-investment/towards-circular-economyen

(9) «In this way, it is possible to question the concepts of proximity, both spatial and social; the governance of flows, including the role of lifestyle and urban practices in material exchanges; and the role of local and territorial stakeholders. To date, this field of interdisciplinary research is fragmentary ».
(10) Loi n 92-646 du 13 juillet 1992 relative à l'élimination des déchets ainsi qu'aux installations classées pour la protection de l'environnement.

(11) Le déchet a été considéré exclusivement comme une ressource jusqu'à la fin du XIXe siècle où il était généralement réinjecté dans l'économie locale (amendement des terres agricoles, recyclage, réutilisation, etc.) (Barles, 2005).

(12) Nous faisons référence au corpus théorique dense des études de la proximité dans le développement économique territorial, notamment les effets de la proximité spatiale dans l'émergence de systèmes productifs locaux (voir notamment : Pecqueur, Zimmermann, 2004).

(13) Ce travail d'identification des flux est la base du projet de recherche MUEED. II fait I'objet d'autres publications (Durand et al., 2016).

(14) L'échantillon constitué visait à un équilibre entre les différents acteurs des déchets (collectivités locales, entreprises prestataires, éco-organismes, secteur de l'économie sociale et solidaire, observatoires régionaux et services de l'État). Les entretiens ont été réalisés uniformément auprès des acteurs des trois terrains étudiés (Rennes, Le Mans, Marne La Vallée), ainsi que d'acteurs nationaux (comme les éco-organismes).

(15) Ces éléments ne peuvent pas être développés ici. Ils feront l'objet de publications différentes.

(16) Lors des entretiens réalisés, chaque acteur peut ainsi percevoir la proximité selon différentes modalités, parfois contradictoires l'une avec l'autre, en fonction du sujet qu'il évoque ou du moment de son intervention.

(17) Ces flux de déchets ont été identifiés par le projet MUEED (Durand et al., 2016).

(18) Ces analyses d'empreinte environnementale, réalisées dans le cadre du projet MUEED, fon l'objet de publications à venir.

(19) Les résultats issus du projet MUEED ont permis de déterminer une empreinte environnementale des filières afin de faire cette différence.

(20) C'est le résultat de la loi NOTRe (Nouvelle organisation territoriale de la République), publiée en août 2015.

(21) La planification relative aux carrières a subi la même évolution que celle des déchets, en passant depuis la loi ALUR publiée en mars 2014, de l'échelle départementale à l'échelle régionale.

(22) cf. Les articles portant sur l'écologie industrielle et territoriale dans ce même numéro thématique de la revue Flux (2017/3-4, № 109-110) : Hampikian; Beaurain, Maillefert et Varlet; Herbelin et Buclet. 


\section{BibliograPhIE}

ADEME, 2014, Économie circulaire : notions, Référent : Alain Geldron.

ARnSPERGer, C., BourG, D., 2016, Vers une économie authentiquement circulaire : Réflexions sur les fondements d'un indicateur de circularité, Revue de l'OFCE, 2016/1, vol. 145, p. 91-125. DOI:10.3917/reof.145.0091

BAHERS J.-B., 2014, Métabolisme territorial et filières de récupération-recyclage: le cas des déchets d'équipements électriques et électroniques (BES) en Midi-Pyrénées, Développement durable. [En ligne], Vol. 5, n¹ | Février 2014. DOI: 10.4000/developpementdurable.10159

Bahers J.-B., Capurso I., Gossart C., 2015, Réseaux et environnement : regards croisés sur les filières de gestion des Déchets d'Équipement Électriques et Électroniques à Toulouse et à Milan, Flux, 2015/1 (№ 99), p. 32-46.

BARLES S., 2005, L'invention des déchets urbains : France 17901970, Seyssel : Champ Vallon.

BARLES S., 2010, Society, energy and materials: the contribution of urban metabolism studies to sustainable urban development issues, Journal of Environmental Planning and Management, $\mathrm{N}^{\circ} 53$, p. 439-455.

Bazin S., Beckerich C., Delaplace M., 2009, les déterminants de l'émergence de filières productives de déconstruction/ recyclage: patrimoines productifs locaux, proximités organisées et/ou proximité ?, Colloque Sixième journée de la proximité. Le temps des débats, Poitiers, 14-16 octobre 2009.

Beaurain C., Brullot S., 2011, L'écologie industrielle comme processus de développement territorial : une lecture par la proximité, Revue d'Economie Régionale \& Urbaine, 2011/2 (avril), p. 313-340. DOI:10.3917/reru.112.0313

Beaurain C., Malleferert M., Lenoir Valet D., 2017, La proximité au cœur des synergies éco-industrielles dunkerquoises, Flux, 2017/3-4 (No 109-110), p.

Berdier C., Deleuil J.-M., 2010, Le système "ville-déchet», une mise en perspective historique, in : Dorier-Apprilli E. (sous la direction de), Ville et environnement, Paris : Sedes, p. 453-466.

BuCLET N., 2011, Écologie industrielle et territoriale : stratégies locales pour un développement durable, Villeneuve-d'Ascq : Presses Universitaires Septentrion.

BuClet N., 2012, Trajectoires institutionnelles et implications citoyennes dans la gestion des déchets ménagers, in Le Bozec A., Keck G., Barles S., Buclet N. (sous la direction de), Que faire de nos déchets ménagers?, Versailles : Quae, p. 61-99.

BuClet N., 2014, L'économie de fonctionnalité entre éco-conception et territoire: une typologie, Développement durable et territoires. [En ligne], Vol. 5, n $1 \mid$ février 2014, DOI:10.4000/developpementdurable.10134

BUCLET N., 2015, Écologie industrielle et économie circulaire, définitions et principes, in : Alix Y., Mat N., Cerceau J. (sous la direction de), Économie circulaire et écosystèmes portuaires, Cormelles-le-Royal : EMS, p. 27-42.

Cireli C, Maccaglia F., 2015, Les politiques publiques des déchets à l'épreuve des contextes locaux: la difficile territorialisation de la proximité, in: Durand M., Djellouli Y.,
Naoarine C., Bertrand J.-R. (sous la direction de), Gestion des déchets : Innovations sociales et territoriales, Rennes : PU Rennes, p. 217-228.

Cirelli C., Maccaglia F., Melé P., 2017, "L'incinérateur est trop près, la poubelle trop loin $»$ : gérer les déchets en régime de proximité, Flux, 2017/3-4 (№ 109-110), p.

Coutard O., 2010, Services urbains : la fin des grands réseaux, in: Levy J.-P., Coutard O. (sous la direction de), ÉCOlogies urbaines, Paris: Economica/Anthropos, Coll. Villes, p. 102-129.

D'ArRAs D., 2008, Les déchets, sur la voie de l'économie circulaire, Annales des Mines - Réalités industrielles, № 2008/4, p. 42-44.

De Oliveira N., 2013, Gestãopública de resíduossólidosurbanos: problemática e práticas de gestão no oesteparanaense, Thèse de doctorat, Curitiba, Universida de Federal do Paraná.

DesvauX P., 2017, Économie circulaire acritique et condition post-politique : analyse de la valorisation des déchets dans I'agglomération lyonnaise, Flux, 2017/2 (№ 108), p. 36-50.

Durand M., Bahers J.-B., Beraud H., 2016, Vers une économie circulaire... de proximité ? Une spatialité à géométrie variable, Déchets, sciences et techniques, $\mathrm{N}^{\circ} 71$, p. 49-63.

Durand M., Bahers J.-B., Bonnierbale T., Beraud H., Barroca B. 2016, Économie circulaire... de proximité ? Métabolisme urbain, empreinte environnementale et politique de gestion des déchets, rapport final, Angers : ADEME.

Durand M., Diellouli Y., NaOarine C., Bertrand J.-R. (sous la direction de), 2015, Gestion des déchets : Innovations sociales et territoriales, Rennes : PU Rennes.

Emelianoff C., 2007, La ville durable: I'hypothèse d'un tournant urbanistique en Europe, L'Information géographique, $N^{\circ} 71$, p. 48.

Fan X., Bourg D., ERKMAN S., 2006, L'économie circulaire en Chine. Vers une prise en compte de l'environnement dans le système économique chinois?, Futuribles, № 324, p. 21-41.

Ghiseluin P., Cialani C., Ulgiati S., 2016, A review on circular economy: The expected transition to a balanced interplay of environmental and economic systems, Journal of Cleaner Production, № 114 , p. 11-32.

Gilly J.-P., Torre A. (sous la direction de), 2000, Dynamiques de proximité, Paris : Éditions L'Harmattan.

GOUHIER J., 2000, Au-delà du déchet, le territoire de qualité, manuel de rudologie, Rouen : PURH.

Gregson N., Crang M., Fuller S., Holmes H., 2015, Interrogating the circular economy: The moral economy of resource recovery in the EU, Economy and Society, $\mathrm{N}^{\circ} 44$, p. 218-243.

HamPIIIAN Z., 2017, Structuration urbaine d'un réseau de chaleur et énergie fatale : la proximité comme variable dynamique, Flux, 2017/3-4 (No 109-110), p.

Herbelin A., Buclet N., 2017, L'agglomération de Roussillon en moyenne vallée du Rhône: une proximité historique remise en cause entre ville et industrie, Flux, 2017/3-4 $\left(\mathrm{N}^{\circ} 109-110\right), \mathrm{p}$ 
Jensen P. D., Basson L., Hellawell E. E., Bailey M. R., Leach M., 2011, Quantifying 'geographic proximity': Experiences from the United Kingdom's national industrial symbiosis program, Resources, Conservation and Recycling, № 55, p. 703-712.

JUNQUA G., BRULlot S., 2015, Écologie industrielle et territoriale : COLEIT 2012, Colloque interdisciplinaire sur l'écologie industrielle et territoriale, Paris : Presses de l'École des Mines.

Matias Mendes M., 2015, Les conflits en aménagement éclairés par la question de la légitimité dans les discours : Le cas de la gouvernance des ISDND en France métropolitaine, Thèse de doctorat en Urbanisme, sous la direction de Jean-Michel Deleuil, Lyon, INSA.

Méry J., Torre A., et gestion des déchets : application à la mise en décharge, Colloque Sixième journée de la proximité. Le temps des débats, Poitiers, 14-16 octobre 2009.

Morris D., 1982, Self Reliant Cities: Energy and the Transformation of Urban America, San Francisco: Random House, Inc.

\section{Résumé - Jean-Baptiste Bahers, Mathieu Durand, Hélène Beraud - Quelle territorialité pour l'éco- nomie circulaire ? Interprétation des typologies de proximité dans la gestion des déchets}

Si l'économie circulaire structure aujourd'hui le débat public autour des questions de maîtrise des flux de matière dans le cadre de la transition socio-écologique, la question de sa territorialité n'est pas véritablement posée publiquement. L'une des façons de comprendre ces enjeux est de cibler l'un des trois grands domaines qui la composent, le plus ancien et le plus structuré : la gestion des déchets. En effet, les logiques spatiales se complexifient, avec comme cadre de référence l'application du "principe de proximité ». Pour autant, rien n'indique ni l'échelle ni les modalités de cette proximité. Elle semble être à géométrie très variable en fonction du type d'acteur qui la prend en charge, du type de déchets et de la structuration des filières. Cet article cherchera donc à comprendre l'opérationnalisation du principe de proximité en fonction des acteurs de la gestion des déchets, afin d'analyser comment ces acteurs s'approprient les enjeux territoriaux de l'économie circulaire.

Mots clés : territorialité, économie circulaire, gestion des déchets, proximités, métabolisme urbain.
Nicourt C., Girault J. M., 2006, L’usage du principe de proximité comme instrument d'ajustement de la décision publique, Développement durable et territoires. [En ligne], Dossier 7 | 2006, DOI:10.4000/developpementdurable.2572

Pecqueur B., Zimmermann J.-B., 2004, Économie de proximités, Paris : Hermès-Lavoisier.

Rocher L., 2006, Gouverner les déchets. Gestion territoriale des déchets ménagers et participation publique, Thèse de doctorat en géographie, sous la direction de Corinne Larrue, Université François Rabelais de Tours.

ROCHer L., 2008, Les contradictions de la gestion intégrée des déchets urbains : I'incinération entre valorisation énergétique et refus social, Flux, 2008/4 ( $\left.N^{\circ} 74\right)$, p. 22-29.

Schiller F., Penn A., Druckman A., Basson L., Royston K., 2014, Exploring Space, Exploiting Opportunities, Journal of Industrial Ecology, № 18, p. 792-798.

Torre A., Beuret. J.-E., 2012, Proximités territoriales, Paris : Economica.

\section{Abstract - Jean-Baptiste Bahers, Mathieu Durand, Hélène Beraud - What Kind of Territoriality for the Circular Economy? An Interpretation of Typologies of Proximity in Waste Management}

While the notion of a circular economy structures public debate regarding socio-ecological transitions of material flows, the issue of its territoriality has not been greatly discussed. One way to understand these issues is to focus the analysis on one of the main and oldest domains: waste management. Indeed, spatial approaches become more complex within the framework of a proximity principle. However, there is no definition of the geographical scale or modalities of this proximity. It is implemented in different ways according to the types of waste, the stakeholders in charge and the organisation of the industrial chain. This article analyses therefore the implementation of proximity, in order to understand how the territorial issues of a circular economy are appropriated.

Keywords: territoriality, circular economy, waste management, proximity, urban metabolism. 